\title{
Stress-ratio-dependent material parameters for improved numerical simulations of textile membrane structures
}

\author{
M. Motevalli* and D. Balzani* \\ * Ruhr University Bochum, Chair of Continuum Mechanics, Universitaetsstr. 150, \\ 44801 Bochum, Germany \\ Email:mehran.motevalli@rub.de,daniel.balzani@rub.de \\ Web page: www.lkm.rub.de
}

\begin{abstract}
A recently proposed orthotropic hyperelastic material model [1-2] for the geometrically nonlinear simulation of woven textile membranes is extended to become the basis of a new approach for structural simulations of improved accuracy. The model is polyconvex and benefits from anisotropic metric tensors in the construction of structural tensors [3]. Anisotropic metric tensors enable various couplings and interactions between principal material directions which are essential in the case of woven fabrics [1]. The proposed nonlinear model is a competitive replacement for the commonly used, however, oversimplified linear elastic formulation. The model contains only 3 effective material properties for glass-PTFE or 4 parameters for PES-PVC fabrics [2]. Following the basic ideas in [4], the nonlinear model parameters can be initially identified and fixed for general groups of fabrics, e.g., glass-PTFE or PES-PVC. For each particular material type, the remaining linear material parameters will be uniquely identified via a convex optimization problem for the given set of experimental data.
\end{abstract}

To improve the accuracy of the model response in structural simulations we propose a new framework to identify and modify material parameters based on the stress ratio locally varying within the structure. To this end, in the first step, the material parameters are separately adjusted to rather classical biaxial tensile tests with load ratios e.g., 1:0.5, 1:1, etc, and further rather uncommon load ratios 1:0.25, 1:0.125, etc. In the second step, an iterative simulation procedure will be applied, where, according to the resulting, locally distributed stress ratio, the corresponding parameters value will be updated at the integration points in the discretized structural problem. For this purpose, suitable interpolation schemes will be applied to identify the parameters at load ratios in between the experimentally considered ones. The iterative scheme will be repeated until the overall parameter change on all points becomes negligible. The efficacy of the proposed method will be assessed by comparing results using the new approach with the results obtained from fixed parameters.

\section{REFERENCES}

[1] M. Motevalli, J. Uhlemann, N. Stranghöner, and D. Balzani, "Geometrically nonlinear simulation of textile membrane structures based on orthotropic hyperelastic energy functions," Composite Structures, vol. 223, 2019.

[2] M. Motevalli, J. Uhlemann, N. Stranghöner, and D. Balzani, "The elastic share of inelastic stressstrain paths of woven fabrics," Materials, vol. 13, 2020.

[3] V. Ebbing, D. Balzani, J. Schröder, P. Neff, and F. Gruttmann, "Construction of anisotropic polyconvex energies and applications to thin shells," Computational Materials Science, vol. 46, no. 3, pp. 639-641, 2009.

[4] L. E. Perotti et al., "Method for the unique identification of hyperelastic material properties using full-field measures. Application to the passive myocardium material response," (eng), International journal for numerical methods in biomedical engineering, vol. 33, no. 11, 2017. 